\title{
Applying the functional independence measure to the assessment of patients with mucopolysaccharidosis
}

\section{Aplicar la medida de independencia funcional a la evaluación de pacientes con mucopolisacaridosis}

\author{
Marcos Almeida Matos ${ }^{1}$, Paloma Silva Lopes ${ }^{1}{ }^{\mathfrak{b}}$, Allan Rodrigues Corsini ${ }^{1}{ }^{\mathfrak{b}}$, Julia \\ Rodi $^{2}$, Chin-To Fong ${ }^{2}$ \\ marcos.almeida@hotmail.com
}

1 Escola Bahiana de Medicina e Saúde Pública, Salvador, Bahia, Brasil, 2 University of Rochester Medical Center, Rochester-NY, USA,

\section{Abstract}

\section{Objective:}

To assess the functional independence of a group of patients with mucopolysaccharidosis using the Functional Independence Measure as a tool that accomplishes this purpose.

\section{Methods:}

This is a cross-sectional study of patients with mucopolysaccharidosis. Our data was collected between June 2015 and July 2016. In addition to history of present illness and physical examination each study participant was asked to answer a questionnaire to specifically evaluate their functional independence using the functional independence measure. the internal consistency of the functional independence measure was assessed using Cronbach's alpha coefficient.

\section{Results:}

Received : 25 Jun 2020

Revised : 11 Aug 2020

Accepted : 11 Sep 2020

Published: 30 Sep 2020

Keywords:

Mucopolysaccharidosis; evaluation; function; independence; validation

Palabras clave:

Mucopolisacaridosis; evaluación; función; independencia; validación

Copyright: () 2020 Universidad del Valle.

We collected data on 20 patients with mucopolysaccharidosis. The average age was 10.8 (8.6713.03) years, the average weight was $23.6(19.91-27.37) \mathrm{kg}$ and the average height was 1 (0.831.17) $\mathrm{m}$. The most prevalent type of mucopolysaccharidosis in the study was type $\mathrm{VI}(\mathrm{n}=14)$. The average total functional independence measure score was 104.4 (97.61-111.19), the average for the mobility domain was $73.50(68.22-78.78)$ and the average for the cognitive function domain was 30.90 (28.68-33.13). The internal consistency of the entire questionnaire was 0.859 , with values of 0.966 for the mobility domain and 0.624 for the cognitive function domain.

\section{Conclusion:}

The lowest functional independence measure scores were obtained in the following sub-domains: self-care, locomotion and cognitive function. The functional independence measure questionnaire demonstrated internal consistency for the evaluation of functional independence in patients with mucopolysaccharidosis, being able to value all the affected sub-domains separately. 
Conflict of Interest:

None

Corresponding author:

Marcos Almeida Matos. Rua Guilhard Muniz, 175, Itaigara, Salvador-Bahia, Brazil, ZIP 41110-810.

E-mail: marcos.almeida@hotmail.com

\section{Resumen}

\section{Objetivo:}

Evaluar la independencia funcional de un grupo de pacientes con mucopolisacaridosis utilizando la Medida de Independencia Funcional como herramienta para lograr este propósito.

\section{Métodos:}

Este es un estudio transversal de pacientes con mucopolisacaridosis. Nuestros datos se recopilaron entre junio de 2015 y julio de 2016. Además de la historia de la enfermedad actual y el examen físico, se pidió a cada participante del estudio que respondiera un cuestionario para evaluar específicamente su independencia funcional utilizando la medida de independencia funcional. la consistencia interna de la medida de independencia funcional se evaluó mediante el coeficiente alfa de Cronbach.

\section{Resultados:}

Recopilamos datos de 20 pacientes con mucopolisacaridosis. La edad promedio fue de 10.8 (8.67-13.03) años, el peso promedio fue de $23.6(19.91-27.37) \mathrm{kg}$ y la altura promedio fue de $1(0.83-1.17) \mathrm{m}$. El tipo de mucopolisacaridosis más prevalente en el estudio fue el tipo VI $(n=14)$. La puntuación media de la medida de independencia funcional total fue $104,4(97,61-111,19)$, la media del dominio de movilidad fue de $73,50(68,22-78,78)$ y la media del dominio de función cognitiva fue $30,90(28,68-33,13)$. La consistencia interna de todo el cuestionario fue de 0,859 , con valores de 0,966 para el dominio de movilidad y 0,624 para el dominio de función cognitiva.

\section{Conclusión:}

Las puntuaciones más bajas de la medida de independencia funcional se obtuvieron en los siguientes subdominios: autocuidado, locomoción y función cognitiva. El cuestionario de medida de independencia funcional demostró consistencia interna para la evaluación de la independencia funcional en pacientes con mucopolisacaridosis, pudiendo valorar todos los subdominios afectados por separado.

\section{Remark}

\section{1)Why was this study conducted?}

This study was conduct in the hope of improving the knowledge about functional independence in patients affected by mucopolysaccharidosis.

\section{2) What were the most relevant results of the study?}

This study gives additional credence to the use of Functional Independence Measure in mucopolysaccharidosis and emphasis the lower independence of MPS patients, particularly in the domains Self-Care, Locomotion and Cognitive Function.

\section{3) What do these results contribute?}

The findings of this study are crucial to the development of preventive and therapeutic protocols that are aimed at give support and improvement of quality of life and treatment to mucopolysaccharidosis. 
Applying the functional independence measure to the assessment of patients with mucopolysaccharidosis

\section{Introduction}

Mucopolysaccharidoses is a group of rare genetic deficiencies in the degradation of glycosaminoglycans ${ }^{1,2,3}$. The resulting accumulation of glycosaminoglycans in lysosomes leads to cellular dysfunction in many tissues, organs and body systems and consequently cause a wide spectrum of chronic sign and, symptoms can be seen, with steady progression that is ultimately life-threatening ${ }^{1,2,3,4}$.

Mucopolysaccharidoses is lysosomal storage disease that can be classified into seven different types according to clinical characteristics, which can be further subtyped according to the specific enzyme deficiency ${ }^{1,2}$. those types are Mucopolysaccharidoses I (Hurler or Scheie disease), Mucopolysaccharidoses II (Hunter disease), Mucopolysaccharidoses III (Sanfilippo disease), Mucopolysaccharidoses VI (Morquio disease), Mucopolysaccharidoses VI (Maroteaux-Lamy disease), Mucopolysaccharidoses VII (Sly disease), and Mucopolysaccharidoses IX ${ }^{1,2}$. All types are very similar with respect to symptoms and progress of the disease. One of the most common manifestations is the so-called dysostosis multiplex, which leads to a wide variety of musculoskeletal problems such as short stature, stiff joints, claw hand deformity, limb deformities, carpal tunnel syndrome (as well as other kinds of nerve compression syndromes), hip dysplasia and cervical instability ${ }^{1,2,5,6}$. In addition, most types of Mucopolysaccharidoses predispose patients to developing concerning neurodevelopmental problems, such as spasticity, developmental delay and regression, cognitive impairment, and aggressive behaviors ${ }^{1,2,5,6}$.

Given all of these manifestations, mucopolysaccharidoses disorders can be highly incapacitating and have the potential to severely worsen the quality of life and the functional independence of affected patients. However, most studies about mucopolysaccharidoses focus primarily on the medical aspects of these conditions ${ }^{1,3,7}$ without seeking to quantify their impact on the subjects' functional status. Only a few studies have evaluated the functional independence of patients with mucopolysaccharidoses using generic or specific instruments ${ }^{8,9,10,11}$, which provided little specificity for these patients. Lastly, not only is there a dearth of studies on the effect of mucopolysaccharidoses on functional independence, but also are required studies dedicated to evaluating and validating the psychometric component of the instruments used in these patients.

The Functional Independence Measure is a widely used tool that is validated for quantifying the extent to which patients require assistance with their activities of daily living ${ }^{13,14}$. It has been also used in two studies on the functional capacity of patients affected by mucopolysaccharidoses type II despite the lack of knowledge about its psychometric characteristics in that situation ${ }^{10,11}$. Functional Independence Measure could be useful for understanding the impact that mucopolysaccharidoses has on patients' level of function and for evaluating and monitoring long term disease progression ${ }^{10,11}$. However, only assessment of the internal consistence of this questionnaire can give more credence to the results of studies related to this subject. The goal of this study is to extend the findings of previous studies by assessing the functional independence of a group of patients with different types of mucopolysaccharidoses using the Functional Independence Measure and evaluate the internal consistence of the instrument.

\section{Materials and Methods}

A descriptive and analytic cross-sectional study was conducted between June 2017 and July 2018 on a group of patients with Mucopolysaccharidoses at the orthopedic outpatient clinic at Hospital Santa Isabel, Salvador, a tertiary-referral hospital for the entire state of Bahia, Brazil. The study is part of a project entitled "Clinical evaluation of the musculoskeletal 
manifestations of mucopolysaccharidosis", which has been approved by the research ethics committee at the Hospital Santa Isabel da Santa Casa de Misericórdia da Bahia, under no 925.989/2014 and CAAE no 38746914.5.0000.5520.

The sample of the study consisted of outpatients seen at the clinic. The inclusion criteria were individuals able to walk independently. According to the inclusion criteria, patients were required to have a biochemical diagnosis of Mucopolysaccharidoses and be above 6 months of age. Patients were eligible for the study if they had received a definitive diagnosis of Mucopolysaccharidoses based on the activity of specific enzymes. Patients of both sexes and any age group were eligible. Taking into account the rarity of the disease, there was no exclusion criterion, and we accepted all available subjects. Informed consent was obtained from every study participant or their guardian. A history and physical exam were performed and participants were given a questionnaire designed specifically to test their functional independence. Social and demographic data was also collected.

The Brazilian version of functional independence measure ${ }^{12,14}$ was used to test functional independence in all study subjects. The functional independence measure instrument is designed for children aged 7 or older, therefore, the pediatric version (WeeFIM) was used for children under 7 years-old. Fortunately, as performed before, numbers of both instruments can be pooled together ${ }^{9,11}$. All of the interviewers were previously trained according to the user manual for the functional independence measure ${ }^{12}$. The questionnaire is divided into four domains (self-care, mobility, communication and cognitive function) and is composed of 18 items, which are each graded on a scale from 1 to 7 . The minimum total score was 18 and maximum total score was 126 . The highest score (126) indicates that the individual can accomplish the task with complete independence. Decreasing scores correspond to decreasing levels of independence, with a score of 18 signifying complete dependence. Scores are added for each sub-domain and these are then added together to make up the total functional independence measure score ${ }^{12-14}$.

The categorical variables were tallied based on frequency and the continuous variables were described by their averages and standard deviation. We presented the total scores for each domain and sub-domain. We also evaluated the internal consistency of the instrument using the Cronbach alpha coefficient to assess each of the domains and the total. This was done using the following criteria, which was put forth by Landis e Koch ${ }^{15} ; 0.5<\alpha=$ unacceptable correlation; $0.6>a \geq 0.5$ = bad correlation; $0.7>a \geq 0.6$ = questionable correlation; $0.8>a \geq$ $0.7=$ acceptable correlation; $0.9>\alpha \geq 0.8=$ good correlation; $\alpha \geq 0.9=$ excellent correlation. We therefore considered a coefficient greater than 0.7 to be clinically significant. We used a $p$ value of 0.05 as a measure of statistical significance for every test.

\section{Results}

The study sample was made up of 20 patients diagnosed with Mucopolysaccharidoses. The majority of the individuals were male (65\%) and the mean age of 10.85 (8.67-13.03) years. There were three patients under 7 years-old in whom it was used the adjusted version of the instrument (WeeFIM). The majority (70\%) were diagnosed with Mucopolysaccharidoses type VI, and the remaining with Mucopolysaccharidoses I (10\%) and Mucopolysaccharidoses II (20\%) (Table 1). The application of the functional independence measure took approximately $22 \mathrm{~min}$ to be performed by a single trained evaluator. The difficulty in interviewing cognitive impaired patients was overcome by using proxy answer. The items seemed to be adequately answered by the parents, and also easy to be verified by the examiner.

Table 2 shows the mean total, domain and subdomain functional independence measure scores in absolute scores and as percentage of total possible scores. Between the two domains, the mobility domain had the lower percentage score $(80.8 \%)$. Within the mobility domain, the 
Table 1. Clinical characteristics of patients with Mucopolysaccharidosis

\begin{tabular}{lc}
\hline Characteristics & Number or mean (\% or CI) \\
\hline Biological sex & $13(65 \%)$ \\
Male & $7(35 \%)$ \\
Female & $10.85(8.67-13.03)$ \\
\hline Age (years) & $23.64(19,91-27,37)$ \\
Weight (kg) & $1.00(0.83-1.17)$ \\
Height (m) & $2(10 \%)$ \\
Type of Mucopolysaccharidosis & $4(20 \%)$ \\
Type I & $14(70 \%)$ \\
Type II & \\
Type VI & \\
\hline
\end{tabular}

CI - confidence interval

self-care subdomain had the lowest score, followed by the locomotion subdomain (Table 2).

Table 3 shows the internal consistency coefficient between the larger domains and the global score for this instrument was considered to have good correlation (0.859) according to Lands and Koch and was also statistically significant (Table 3). The internal consistency for all of the subdomains that make up the functional independence measure was measured by the alpha Cronbach Index, and was also considered nearly perfect (0.831) and significant when compared to the global score. However, when we compared the items with the subdomains in the instrument, two sub-domains had correlations below the value considered to be clinically satisfactory; namely, the Sphincter Control subdomain (0.142) and the Communication subdomain (0.343). Other data can be found in Table 3.

\section{Discussion}

Our findings show that patients with Mucopolysaccharidoses have lower functional independence scores; about $17 \%$ less than the maximum score on the functional independence measure. The lowest scores were obtained in the Self-care and Locomotion subdomains; which, as a result, led to a decline in the Mobility domain. The internal consistency scores for the functional independence measure (including items and subdomains) demonstrated consistencies ranging from acceptable to excellent. The subdomains compared to the domains (specifically Cognitive Function and Mobility) demonstrated an acceptable internal consistency (greater than 0.7). When the domains were compared to the total functional independence measure score, the results for Mobility were excellent but the results for Cognitive Function were questionable (below 0.7 ). When the total score was compared to the subdomains, most were good or excellent, but the Sphincter Control and Communication subdomains were found to have an unacceptable internal consistency.

We believe that the low scores obtained in the Self-care and Locomotion subdomains

Table 2. Functional independence measure scores for each domain and subdomain in patients with Mucopolysaccharidosis

\begin{tabular}{lcr}
\hline Domains & Mean score (CI) & Mean percentage* \\
\hline FIM Total & $104.40(97.61-111.19)$ & 82.9 \\
\hline Domains & $73.50(68.22-78.78)$ & 80.8 \\
Mobility Domain & $30.90(28.68-33.13)$ & 88.3 \\
Cognitive Function Domain & & \\
Subdomains & $29.65(27.26-32.04)$ & 70.6 \\
Self-care & $13.40(12.83-13.97)$ & 95.7 \\
Sphincter Control & $19.40(17.57-21.26)$ & 92.3 \\
Mobility & $11.05(9.26-12.84)$ & $82.0 / 14.0$ \\
Locomotion & $12.80(11.82-13.78)$ & 91.4 \\
Communication & $18.10(16.53-19.67)$ & 86.2 \\
Cognition & & \\
\hline
\end{tabular}


Table 3. Alpha Cronbach coefficients for each functional independence measure domain and subdomain for patients with mucopolysaccharidosis

\begin{tabular}{lcc}
\hline Domains & FIM domain- total Cronbach $(\boldsymbol{p})$ & FIM subdomain- domain Cronbach $(\boldsymbol{p})$ \\
\hline Mobility & $0.966(<0.001)$ & $0.761(<0.001)$ \\
Cognitive Function & $0.624(<0.001)$ & $0.787(<0.001)$ \\
Global FIM & $0.859(<0.001)$ & FIM item-subdomain Cronbach $(\boldsymbol{p})$ \\
Subdomains & FIM subdomain- total Cronbach $(\boldsymbol{p})$ & $0.787(<0.001)$ \\
Self-care & $0.849(<0.001)$ & $0.937(<0.001)$ \\
Sphincter Control & $0.142(0.552)$ & $0.885(<0.001)$ \\
Mobility & $0.798(<0.001)$ & $0.928(<0.001)$ \\
Locomotion & $0.829(<0.001)$ & $0.914(<0.001)$ \\
Communication & $0.343(0.344)$ & $0.823(<0.001)$ \\
Cognitive Function & $0.873(<0.001)$ & $0.754(<0.001)$ \\
Global Cronbach alpha index & $0.831(<0.001)$ &
\end{tabular}

FIM: functional independence measure

are due to the clinically significant osteoarticular pathologies associated with

Mucopolysaccharidoses ${ }^{1,2,6}$. Patients with Mucopolysaccharidoses present with musculoskeletal rigidity and articular deformities that impair mobility and locomotion 1,2,6,16,17. There are also changes to the vertebral column characterized by kyphosis and scoliosis, and thoracolumbar vertebral rotation is a common finding. These changes lead to difficulty with gait and mobility in patients with Mucopolysaccharidoses and impact their activities of daily living, such as getting out of bed, going to the bathroom, walking and running ${ }^{1,2,6,16,17}$. Apart from these osteoarticular deformities, individuals with Mucopolysaccharidoses also have impaired cardiac, pulmonary and respiratory function and expend more energy in initiating and maintaining gait, which results in early fatigue ${ }^{16,17}$.

Starting in the first few years of life, individuals with Mucopolysaccharidoses demonstrate restricted range of motion, especially in their elbows, knees and hips. This is secondary to involvement of the metaphysis as well as thickening and fibrosis of the articular capsule ${ }^{1,2,6,17}$. These alterations are direct consequences of the accumulation of glycosaminoglycans in musculoskeletal tissues, such as tendons and synovial membranes. The progression of these clinical manifestations depends on the severity and chronicity of the disease, as glycosaminoglycans tends to accumulate in various systems as the individual ages ${ }^{1,2,6,17}$. However, in our sample, most study participants were pediatric patients and did not yet require assistance with walking or standing. This indicates that self-care and locomotion may be even more severely impaired in older patients.

Individuals with Mucopolysaccharidoses present with flexion contractures of the digits (claw hand deformity), median nerve compression (carpal tunnel syndrome) and Dupuytren's contractures ${ }^{18,19}$. As a result, they have a limited ability to perform a pincer grasp and have difficulty holding objects that are important for daily activities, such as knives, forks and toothbrushes and struggle to open faucets or hold soap ${ }^{18,19}$. In addition, the abnormal spacing between their teeth and dental dysplasia impair mastication and deglutition, resulting in dysphagia ${ }^{2,6}$. These factors certainly impact self-care and specific techniques or tools are often required, or even assistance from others, for patients to accomplish daily tasks. Food consistency may need to be altered, as thick pasty foods facilitate the creation of a bolus and help prevent aspiration, choking or dietary deprivation.

Individuals with Mucopolysaccharidoses vary in their cognitive function. Typically, cognitive impairment occurs in Mucopolysaccharidoses types I, II and III 1,2,6. However, we evaluated cognitive function in patients with many different types of Mucopolysaccharidoses in this study and the most prevalent type was type VI, which is thought to have the least association with cognitive impairment. We believe this explains why cognitive function, and especially the communication domain, showed less evidence of impairment in our sample. However, 
even in the absence of significant neurocognitive problems, it is possible that there was still some degree of limitation in the ability to communicate because of factors such as physical dependence, macroglossia and hearing loss, which can all result in the need for assistance with comprehension, self-expression and social interactions.

Although there is no study that assess the value of applying questionnaires to evaluate functional independence in patients with Mucopolysaccharidoses, a few studies have shown a reduction in functional scores in these patients ${ }^{8,11}$. A study conducted by Guarany et al. ${ }^{10}$, made use of the functional independence measure and PEDI (Pediatric Quality of Life Questionnaire) to assess the level of function of 21 patients with Mucopolysaccharidoses. The functional independence measure was answered by a total of 14 participants and 8 of them had global scores below 100 points; only 6 participants achieved a maximum score in the Cognitive Function domain, while only 1 achieved the maximum score in the Mobility domain (10 were below 90 points) ${ }^{10}$; there was also a significant correlation between the functional independence measure and the amplitude of articular movements, which suggests that both measures may be useful markers for progression of neurological aspects of the disease ${ }^{10}$.

Kato et al. ${ }^{11}$, also applied the functional independence measure to evaluate the activities of daily living of 27 patients with Mucopolysaccharidoses type II. Individuals with more severe manifestations of the disease had lower scores than individuals with milder forms; the patients with severe forms of Mucopolysaccharidoses type II had a total score of 43.9 points (34.8\% below the maximum possible score), with Mobility scores of 36.5 (40.1\%) and Cognitive Function scores of 7.4 (21.1\%). Patients with milder forms of Mucopolysaccharidoses II scored a total of 117.7 points (93.4\%) in the total, 85.8 (94.3\%) in Mobility and 31.8 (90.8\%) in Cognitive Function ${ }^{11}$. Mucopolysaccharidoses types I and II are considered to be the most severe types of Mucopolysaccharidoses and it is possible that our study found less evidence of impairment using the functional independence measure because we included less aggressive types of Mucopolysaccharidoses in our study (our results were; Total 104.4; Mobility 73.5 and Cognitive Function 30.9).

The Kato et al. ${ }^{11}$, also found a moderate correlation between the functional independence measure and amplitude of movement in patients with severe disease, but the correlation was weak in patients with milder disease. The authors suggest that the functional independence measure is a new tool that is useful for the evaluation of functional independence in patients with Mucopolysaccharidoses, since it was found to be adequate in measuring the state of the disease from a somatic point of view (mobility) and a mental one (cognitive function) ${ }^{(11}$. However, this study did not make use of the psychometric properties of the functional independence measure questionnaire.

In regards to the internal consistency of the functional independence measure, our study showed that the Cronbach coefficients for the relationship between the domains and subdomains and between the subdomains and the items were greater than the value required for clinical significance (greater than 0.7); however, the Cognitive Function, Sphincter Control and Communication subdomains displayed values below the accepted threshold for clinical significance. Given these findings, it did not seem appropriate to add the scores for these disparate subdomains, since they are affected differently by the disease. We therefore propose that the functional independence measure scores be presented and analyzed separately by domain and subdomain, as it is not possible to use the global score to differentiate between patients of different Mucopolysaccharidoses types.

Hobart et al. ${ }^{20}$, evaluated the internal consistency of the functional independence measure when applied to patents with diverse neurological conditions and established that it was internally consistent based on the Cronbach coefficient. Their results were similar to ours; the coefficients they calculated were as follows; 0.87 for the comparison between the total and 
the items, 0.60 for the Mobility domain and 0.63 for the Cognitive Function domain. When confronted with the value of the items, the internal consistency was found to be 0.95 for the total functional independence measure score, 0.95 for the Mobility domain and 0.89 for the Cognitive Function domain ${ }^{20}$. Not only are these results similar to ours, they also support the recommendation to present and analyze the functional independence measure based on separate scores for the subdomains and the domains and provide evidence against the use of the total score in isolation.

The main limitations of this study were the small sample size for Mucopolysaccharidoses types I and II, but this is one of the constraints in studies on rare genetic disorders. On the other hand, our choice to evaluate multiple types of Mucopolysaccharidoses means that our data is better at reflecting the broad impact of these diseases on functional independence instead of focusing on one specific type. Despite this study's limitations, it nonetheless contributes to our understanding of the degree to which patients with Mucopolysaccharidoses require assistance with (and face impairments in) their mental and physical health, especially given the dearth of information on the topic. In addition, using the functional independence measure instead of a specific questionnaire enables us to compare our results to those obtained in other similar diseases and in the general population, which strengthens the study.

\section{Conclusions}

Patients with Mucopolysaccharidoses have scores lower than the maximum functional independence measure scores in all of the domains and subdomains that we analyzed, with the Self-care, Locomotion and Cognitive Function subdomains representing the lowest scores. The functional independence measure questionnaire demonstrated internal consistency when applied to the evaluation of functional independence in patients with Mucopolysaccharidoses. The following coefficients were obtained; 0.859 for the total, 0.624 for the Mobility domain and 0.966 for the Cognitive Function domain. However, the authors recommend that the results for the functional independence measure be analyzed by looking at the subdomains, since the correlations obtained were superior to those found for the domains.

\section{References}

1. Vairo F, Federhen A, Baldo G, Riegel M, Burin M, Leistner-Segal S, et al. Diagnostic and treatment strategies in mucopolysaccharidosis VI. Appl Clin Genet. 2015; 8: 245-55. doi: 10.2147/TACG.S68650.

2. Wolf DA, Banerjee S, Hackett PB, Whitley CB, Mclvor RS, Low WC. Gene therapy for neurologic manifestations of mucopolysaccharidoses. Expert Opin Drug Deliv. 2015; 12(2): 283-296. doi: 10.1517/17425247.2015.966682.

3. Guffon N, Heron B, Chabrol B, Feillet F, Montauban V, Valayannopoulos V. Diagnosis, quality of life, and treatment of patients with Hunter syndrome in the French healthcare system: a retrospective observational study. Orphanet J Rare Dis. 2015; 10: 43. doi: 10.1186/s13023-015-0259-0.

4. Guarany NR, Vanz AP, Wilke MVMB, Bender DD, Borges MD, Giugliani R, et al. Mucopolysaccharidosis: caregiver quality of life. J Inborn Errors Metabolism Screening. 2016; 3: 1-7. doi: 10.1177/2326409815613804

5. Needham M, Packman W, Quinn N. Health-related quality of life in patients with MPS II. J Genet Couns. 2015; 24: 635-644. doi: 10.1007/s10897-014-9791-7.

6. Morishita K, Petty RE. Musculoskeletal manifestations of mucopolysaccharidoses. Rheumatology. 2011; 50: 19-25. doi: 10.1093/rheumatology/ker397.

7. Raluy-Callado M, Chen WH, Whiteman DA, Fang J, Wiklund I. The impact of Hunter syndrome 
Applying the functional independence measure to the assessment of patients with mucopolysaccharidosis

(mucopolysaccharidosis type II) on health-related quality of life. Orphanet J Rare Dis. 2013; 8: 101. doi: 10.1186/1750-1172-8-101.

8. Wiklund I, Raluy-Callado M, Stull DE, Jangelind Y, Whiteman DAH, Chen WH. The Hunter SyndromeFunctional outcomes for Clinical Understanding Scale (HS-FOCUS) Questionnaire: evaluation of measurement properties. Qual Life Res. 2013; 22: 875-884. doi: 10.1007/s11136-012-0196-5.

9. Wiklund I, Raluy-Callado M, Chen WH, Muenzer J, Fang J, Whiteman D. The Hunter Syndrome-Functional outcomes for Clinical Understanding Scale (HS-FOCUS) Questionnaire: item reduction and further validation. Qual Life Res. 2014; 23: 2457-2462. doi: 10.1007/s11136-014-0703-y.

10. Guarany NG, Schwartzb IVD, Guarany FC, Giugliania R. Functional capacity evaluation of patients with mucopolysaccharidosis. J Ped Rehab Med. 2012; 5: 37-46. doi: 10.3233/PRM-2012-0194.

11. Kato T, Kato Z, Kuratsubo I, Ota T, Orii T, Kondo N, Suzuki Y. Evaluation of ADL in patients with Hunter disease using FIM score. Brain Development 2007; 29: 298-305. doi: 10.1016/j.braindev.2006.08.015.

12. Riberto M. Orientação funcional para utilização da MIF: medida de independência funcional (MIF para adultos). Brasil: HC-FMUSP-DMR. 21p.

13. Riberto M, Miyazaki MH; Jucá SSH, Sakamato H, Pinto PPN, Battistella LR. Validação da versão brasileira da Medida de Independência. Funcional Acta Fisiatr. 2004;11(2): 72-76. doi: 10.5935/0104-7795.20040003.

14. Keith RA, Granger CV, Hamilton BB, Sherwin FS. The functional independence measure: a new tool for rehabilitation. Adv Clin Rehabil. 1987; 1: 6-18. PMID: 3503663.

15. Landis JR, Koch GG. The measurement of observer agreement for categorical data. Biometrics. 1977; 33 159-174. PMID: 843571.

16. Matos MA, Prado A, Schenkel G, Barreto R, Acosta AX. Energy expenditure during gait in patients with mucopolysaccharidosis. Acta Ortop Bras. 2013; 21(2): 116-9. doi: 10.1590/S1413-78522013000200009.

17. Matos MA, Barreto R, Acosta AX. Evaluation of motor response in mucopolysaccharidosis patients treated with enzyme replacement therapy. Ortop Traumatol Rehabil. 2013; 15(5): 389-93. doi: $10.5604 / 15093492.1084240$.

18. Haddad FS, Jones DH, Vellodi A, Kane N, Pitt MC. Carpal tunnel syndrome in the mucopolysaccharidoses and mucolipidoses. J Bone Joint Surg Br. 1997; 79(4): 576-82. doi: 10.1302/0301-620x.79b4.7547.

19. Aslam R, Hendriksz CJ, Jester A. Subjective and objective assessment of hand function in mucopolysaccharidosis IVa Patients. JIMD Rep. 2013; 9: 59-65. doi: 10.1007/8904_2012_179.

20. Hobart JC, Lamping DL, Freeman JA, Langdon DW, McLellan DL, Greenwood RJ, Thompson AJ. Evidence-based measurement: which disability scale for neurologic rehabilitation? Neurology. $2001 ; 57(4)$ : 63944. doi: 10.1212/wnl.57.4.639. 